\title{
Pressure Groups and Experts in Environmental Regulation*
}

\author{
Nicolás Porteiro ${ }^{\dagger}$
}

July 7,2001

\begin{abstract}
We study a problem of adverse selection in the context of environmental regulation, where the firm may suffer from a certain degree of ignorance about its own type. In this framework we analyze the impact on the regulatory process of the presence of informed third-parties. We study and compare the role of environmentalists and of unbiased experts. Our analysis allows us to support the spirit of the actual E.U. legislation concerning the assessment of the impact of public and private projects on the environment, and provides insights about the potential role of interested parties as information providers in a regulatory process.
\end{abstract}

JEL Classification: D82, L51.

Keywords: environmental regulation, ignorance, environmentalists, experts.

${ }^{*}$ I am very grateful to Inés Macho-Stadler for her help and support. I also would like to thank Johan Eyckmans, Paula González, Pau Olivella, David Pérez-Castrillo and the participants in the XVI Jornadas de Economía Industrial (Madrid) for their helpful comments. Financial support from the Generalitat de Catalunya (grant 1998FI 00020) and from the Spanish Ministry of Education (project BEC2000-0172) is gratefully acknowledged. All remaining errors are mine.

†Department d'Economia i Història Econòmica. Universitat Autònoma de Barcelona. Bellaterra 08193 (Barcelona). Spain. Phone: (34) 93581 1811. Fax: (34) 93581 2012. E-mail: porteiro@idea.uab.es 


\section{Introduction}

The use of incentive-based regulatory mechanisms to deal with environmental problems has received increasing attention in the literature over the last years. The standard model presents one (or several) agents who posses relevant private information concerning either their private costs or the effect of their performance on the environment. ${ }^{1}$ We extend this standard model in two closely related ways. First, in those situations where the informational advantage concerns the environmental impact of an economic activity, and not an intrinsic cost parameter, the standard model can be unrealistic. Since the source of the informative advantage with respect to the regulator is extrinsic to the firm, this advantage may not be perfect and the firms may need to engage in costly searching to gather information concerning their type. Second, another important characteristic of environmental problems that has usually not been captured in the literature is the existence of pressure groups, such as the environmentalists, who may have access to some relevant information, and who have strong incentives in affecting the regulatory process. In this respect, Lewis (1996), when presenting the areas of future research in environmental regulation, raises as an important issue the development of strategies for information acquisition and specifically considers the role of interested parties with stakes in the regulation, as potential information providers.

The aim of this paper is to develop a model of environmental regulation under adverse selection where we study the implications of the existence of ignorance, understood as the possibility that the firm is not sure about its type when signing the contract. We also analyze the impact of the presence of third-parties with access to relevant information. We consider the regulation of a firm that is chosen to undertake a public project with a fixed social value, but that generates environmental damage. ${ }^{2}$ We assume that the information available to the firm as to the type of project it is endowed with (highly damaging or not), is not perfect.

We first consider the design of the optimal regulatory contract in the absence of informed third-parties. We show that there exists a positive lower bound in the degree of ignorance, from which on the contract is pooling for those firms who do not know the type of project they are endowed with (the ignorants) and those who have a highly damaging project (the inefficient firms). We then introduce a potentially informed third-party who can costly gather some relevant information about the firm's type. We analyze which is the best position for this party in the timing, from the principal's perspective. In other words, we study whether it is more efficient to ask him to reveal his information at the beginning of the regulatory process (using him as a filter) or at the end (using him as a check to threaten those firms who misreport their type). We independently study two different kinds of parties. On the one hand, we analyze the impact of informed environmentalists, that we assume to only care about environmental quality. Therefore, they will only disclose their information if it leads to a lower level of pollution. On

\footnotetext{
${ }^{1}$ For a general overview of these models see Lewis (1996).

${ }^{2}$ A similar starting point was used by Boyer and Laffont (1999). They develop a study on the optimal instrument choice for environmental regulation, but their aim is completely different from ours since they focus on an incomplete contract approach to political economy.
} 
the other hand, we also present the situation where the regulator has the possibility of using an unbiased expert who has access to a certain degree of information, and who will always be willing to disclose it. In both configurations, we show that the position of third-party has no direct effect on the contract. That is, in this setting their optimal location in the timing is only determined by the overall costs of gathering information.

We finally identify when does the game with experts dominate that with environmentalists. Not surprisingly, we find that there is a threshold level for the cost of raising public funds, from which on it is not worth using experts (they have to be compensated for the searching costs in which they incur). More surprisingly, we find that for relatively low levels of ignorance, by using experts instead of environmentalists, we have no allocative efficiency gain. That is, the contracts in the two cases are equivalent. Even if the environmentalists do not disclose all the information they have, the optimal contract with experts and with environmentalists is the same, and hence the overall "relevant" information in the system is unaltered. The reason is that for low levels of ignorance, the contract is pooling for two types of firms (the ignorant and the inefficient). The difference in the information disclosure affects only this part of the population leading, therefore, to the same contract.

We first perform all the analysis when the level of ignorance is exogenously fixed. In the last part of the paper we consider that the degree of ignorance is a choice variable of the firm. For the type of problem we are dealing with, it is natural to assume that the firm's decision on its level of information acquisition is unobservable for the principal. Consequently, we develop our analysis for an information gathering decision that is simultaneous to the design of the contract. We find that the regulator's choice on the level of information is more extreme than the firm's. If the firm's decision is to acquire a low degree of information, the regulator would choose a lower level. Conversely, if the firm chooses to be well informed, the principal's choice of information would be even higher.

The timing selection with endogenous ignorance has to take into account the bias in the firm's choice of information with respect to the social optimum, as well as the incentives the alternative timings give for the firm's information gathering.

The analysis we perform has some policy implications that are worth noting. First, we can interpret the problem on the selection of the optimal timing, as a choice of who should take the initiative in the assessment of the impact of the project. A timing with the third-parties placed at the end of the process gives the firm the initiative in the assessment, while a timing with the third parties as a filter corresponds to a situation in which the public powers take this initiative. Our results support the second alternative as optimal, provided the extra cost of public funds is not too high. This goes in favor of the existing E.U. legislation concerning the assessment of the impact of public and private projects on the environment.

Our analysis can also be a useful starting point for the study of the implications of relying on interested parties' information. It provides some insights about the benefits (acquisition of information at a lower costs) and the drawbacks (the bias that their selfish motivation places on the information disclosure) of using an interested party to acquire information. The design 
of future policies could be improved if the policymaker profited from the information acquired by agents with personal interests in the field.

In the literature, the possibility of facing an imperfectly informed agent was first addressed by Lewis and Sappington (1993) in the context of a general adverse selection problem. There, they construct the optimal contract for an agent who suffers from an exogenous level of ignorance. This ignorance is present in the form of a given probability that the firm shares only the same imperfect information as the principal. The resulting contract differs widely from the standard adverse selection one, since pooling and discontinuities appear. More recently, Kessler (1998) introduces the possibility of endogeneizing the level of ignorance, and shows that the agent will never be interested in being perfectly informed, and therefore, that the lack of information has a certain strategic value. Contrary to our approach, Kessler's analysis is performed in a sequential setting in which the firm's decision to acquire information is prior to the design of the contract. However, she does not study the relation among the incentives of the principal and those of the firm, and does not include in her analysis the presence of informed third-parties. In a similar setting, and also in a two states of nature framework, Crémer and Khalil (1994), construct the optimal contract that a principal would offer to an agent that is either perfectly informed or not informed at all about the realization of the random variable. ${ }^{3}$

Milgrom and Roberts (1986), develop an interesting analysis of how a decisionmaker can benefit from the information of interested parties. Their model focuses on the design of optimal information acquisition strategies and hence, takes a more mechanism design approach than our work. Moreover, their results can not be directly applied in our context since the interested party in our game is only perfectly informed (contrary to their framework), and may actually have no relevant information available for the principal.

The remaining of the paper is as follows: Section 2 introduces the model. Section 3 develops the problem of an exogenous and partial level of ignorance. Section 4 analyzes the impact of the presence of informed third parties (environmentalists and experts). Section 5 investigates the consequences of endogeneizing the level of ignorance. Finally, Section 6 briefly concludes and elaborates on the policy implications of the analysis performed. All proofs are in the Appendix.

\section{The Model}

We consider a regulator who delegates the realization of a public project to a firm. Let $S$ represent the social value of the project, which for simplicity is assumed to be large enough to make the realization always desirable. Its implementation generates environmental damage according to the function $V(\alpha, e)$, where $e$ is the effort exerted by the firm in order to preserve the environment from damage (unobservable for the principal), and $\alpha$ represents the type of project. The higher is the value of $\alpha$ the more effect has the firm's effort in reducing the environmental impact of the project.

We assume that $V(\alpha, e)$ is ex-post verifiable as a whole, but not its components. This means

\footnotetext{
${ }^{3}$ The same analysis with more than two states of nature is performed in Crémer and Khalil (1998).
} 
that once the project is finished, the principal can evaluate the overall amount of damage that it caused, but he cannot infer whether the pollution came from a bad project or a firm exerting a low effort.

In order to be able to derive explicit solutions, we consider the specific "damage" function $V(\alpha, e)=D-\alpha e$, with $D$ being a given constant. We assume that $\alpha$ can take two values, and its domain is $\alpha \in\{\underline{\alpha}, \bar{\alpha}\}$, with $\underline{\alpha}=1$. This construction allows us to discuss some comparative statics in terms of the relative advantage of one project over the other as a function of $\bar{\alpha}$. Moreover we take the value of $\bar{\alpha}$ as belonging to $(1,2) .{ }^{4}$ The prior probability that the project is efficient is $v=\operatorname{Pr}(\alpha=\bar{\alpha})$. Hence, the expected efficiency parameter of the project is:

$$
\hat{\alpha}=E(\alpha)=v \bar{\alpha}+(1-v)=1+v(\bar{\alpha}-1) .
$$

Notice that, with this construction, ex-ante there are not two types of firms, but two types of projects. The problem becomes an adverse selection one only if the firm (and not the regulator) gets to know the type of project it is entitled with, before the contract is signed. Then, a firm that knows its project is efficient becomes a "low-polluting" firm, and if it knows it is inefficient, it becomes a "high-polluting" one. In other words, even if we consider an environmental problem where the source of the informational problem is extrinsic to the firm (and not a private technological parameter), we refer to a firm by the type of project it is endowed with.

The cost of the pollution abatement effort of the firm is given by $\Psi(e)$, with $\Psi^{\prime}>0, \Psi^{\prime \prime} \geq 0$, $\Psi^{\prime \prime \prime} \geq 0 . .^{5}$

We denote by $t$ the transfer to the regulated firm. The public funds expended by the regulator have a marginal cost $1+\lambda$, with $\lambda>0$, due to the distortionary impact of taxation in the economy. ${ }^{6}$ According to this, the consumers' welfare is:

$$
C S=S-V(\alpha, e)-(1+\lambda) t
$$

The firm's profits are:

$$
U=t-\Psi(e)
$$

The social welfare, given by the sum of the consumers' surplus and the firm's profits is:

$$
W=C S+U=S-V(\alpha, e)-(1+\lambda) \Psi(e)-\lambda U .
$$

Finally, note that we have constructed the model assuming that the social planner puts equal weight to the consumers' surplus and the profits of the firm in the objective function, but that

\footnotetext{
${ }^{4}$ We restrict the domain of $\bar{\alpha}$ in order to avoid dealing with degenerated situations like having an extremely efficient project (very high value of $\bar{\alpha}$ ). With the domain chosen here the "good" project is never more than twice as efficient as the "bad" one.

${ }^{5}$ The assumption $\Psi^{\prime \prime \prime} \geq 0$ is usual in this models to ensure that the regulator's objective function is concave and that optimal contract is deterministic. See, for instance, Laffont and Tirole (1993) page 35.

${ }^{6}$ The inclusion of a distortion $\lambda>0$ is usual in regulatory problems. It is also supported by stylized facts, according to Jones, Tandon and Vogelsand (1990), the mean value of $\lambda$ for the developed countries can be considered of the order of 0.3 , and even higher in developing ones.
} 
due to the extra costs of public funds $(\lambda)$, eventually he wants to make the firm's rent the smallest possible. An alternative construction is to assume that the public expenditures are not penalized, but that the consumers' surplus has a higher weight than the firm's profits in the objective function. This would generate a very similar model. In particular, the regulator would still be interested in minimizing the firm's informational rents.

\section{Regulation under Exogenous Partial Ignorance}

In this section we present a situation where the only players involved are the regulator and the firm and in which the firm has some degree of informational advantage over the regulator, but this advantage is not perfect. We assume that the firm knows the type of project only with a certain probability, that by now, we take as exogenously given. We model it by considering that the firm, before signing the contract improves its informational status by means of a signal $s^{F}$, defined as follows:

$$
\begin{aligned}
& s^{F}=\{\alpha\} \text { with probability } x \\
& s^{F}=\{\varnothing\} \text { with probability } 1-x .
\end{aligned}
$$

As we are dealing with an exogenous level of ignorance, the firm takes the value of $x$ as given and we ignore the cost of this level of information: either the signal was costless, or if costly it was purchased before the starting point of our analysis.

The informational structure determines the existence of three types of firms: the ones that know that their project is efficient, the ones that know it is inefficient, and the ones who do not know the type of project they are endowed with. We will refer to them as the efficient type, the inefficient type and the ignorants.

The objective function of the regulator under partial ignorance $\left(W^{I}\right)$ is the following:

$$
\begin{aligned}
E W= & (1-x)\left(S-V\left(\widehat{\alpha}, e^{I}\right)-(1+\lambda) \Psi\left(e^{I}\right)-\lambda U^{I}\right)+ \\
& x[v(S-V(\bar{\alpha}, \bar{e})-(1+\lambda) \Psi(\bar{e})-\lambda \bar{U})+(1-v)(S-V(1, \underline{e})-(1+\lambda) \Psi(\underline{e})-\lambda \underline{U})] .
\end{aligned}
$$

The first-best choice of the regulator if it did not suffered from asymmetric information, would be given by a reimbursement of the costs $(t=\Psi(e))$ where the optimal effort $e$ is the one that equates the marginal cost with the expected marginal reduction in environmental damage. If both, the firm and the regulator, suffered from full ignorance, the optimal level of effort would be determined by the expected type of project $(\widehat{\alpha})$.

Assume now that the type of the firm is unknown. The fact that $V(\alpha, e)$ is ex-post verifiable implies that the principal can commit to severely punish any firm signing a contract that expost is revealed unfulfilled. That is, the firm will only dare to exert a level of effort different to the one written in the contract if it is sure about the resulting environmental damage. Hence, the ex-post verifiability of $V(\cdot)$ eliminates any possibility of strategic behavior, either by the ignorant, or by the inefficient firms. The strategic behavior is reduced to the fact that efficient 
firms can deviate by pretending its project is bad and also by pretending they do not know the value of $\alpha$.

The optimal menu of contracts has to include an effort choice and a transfer for each type of firm. Note that if the probability that the firm learns its type $(x)$ goes to one we have the classical adverse selection problem; while when $x$ tends to zero we have a world with complete uncertainty about the type of project. Let $\Phi(e) \equiv \Psi(e)-\Psi\left(\frac{e}{\bar{\alpha}}\right)$. Lemma 1 summarizes the characteristics of the optimal menu of contracts.

Lemma 1 The optimal menu of contracts under partial ignorance is:

1. For the high type:

$$
\begin{gathered}
\bar{e} \text { is s.t. } \Psi^{\prime}(\bar{e})=\frac{\bar{\alpha}}{1+\lambda} \\
\bar{t}=\Psi(\bar{e})+\Phi\left(e^{I}\right) .
\end{gathered}
$$

2. For the low and the ignorance types:

$$
\underline{t}=\Psi(\underline{e}), t^{I}=\Psi\left(e^{I}\right)
$$

where, if $x \leq \bar{x}$, with $\bar{x}=\frac{\bar{\alpha}-1}{\bar{\alpha}-1+\lambda \Phi^{\prime}(\underline{e})}<1$, then:

$$
\begin{gathered}
\underline{e} \text { is s.t. } \Psi^{\prime}(\underline{e})=\frac{1}{1+\lambda} \\
e^{I} \text { is s.t. } \Psi^{\prime}\left(e^{I}\right)=\frac{\widehat{\alpha}}{1+\lambda}-\frac{x v}{1-x} \frac{\lambda}{1+\lambda} \Phi^{\prime}\left(e^{I}\right) .
\end{gathered}
$$

If $x>\bar{x}$, then:

$$
e^{I} \text { and } \underline{e} \text { are s.t. } \Psi^{\prime}\left(e^{I}\right)=\frac{1}{1+\lambda}\left(\frac{(1-v) x+\widehat{\alpha}(1-x)}{1-x v}\right)-\frac{x v}{1-x v} \frac{\lambda}{1+\lambda} \Phi^{\prime}\left(e^{I}\right) .
$$

To understand the result stated in Lemma 1 let us discuss first the extreme cases. Under full ignorance $(x=0)$ the solution does not give any informational rents to the firm, as it does not have an informational advantage. When $x=1$, we have the traditional adverse selection contract, the efficient firm's pollution is unaltered (non-distortion at the top), the inefficient firm's pollution is increased, its effort is lowered to avoid giving too much rent to the efficient one. The distortion is increasing on the proportion of efficient projects $(v)$, on the social cost of public funds $(\lambda)$, and on the technological gap, in other words, the better the firm's project, the tougher the contract.

The comparison of $x=0$ and $x=1$ shows that the presence of ignorance has two opposite effects on the efficiency of the contract. On the one hand, there is an allocative inefficiency, because the firm is offered a pooling contract that is dominated by the separating one in which the effort is chosen contingent on the project's efficiency. On the other hand, however, the principal has also a gain from this contract, the firm's lack of information eliminates its power 
to obtain informational rents. That is, dealing with an informed agent is better from the perspective of the accuracy of the contract, but it is costly in terms of public expenditures. ${ }^{7}$

Now consider the case $x \in(0,1)$. The only strategic agent is the efficient firm. The value of its information, i.e., the informational rent of the efficient firm is increasing on the level of effort $\left(\Phi^{\prime}(e)>0\right)$. Hence the best deviation is signing the contract with the highest associated value of $e$. In the symmetric information contract, $e^{I}>\underline{e}$, because $e^{I}$ is constructed for the expected efficiency level. Therefore, the regulator can start by distorting downwards only the ignorance contract, as this contract is the most appealing for the firm. This distortion is increasing in $x$ since it is a measure of the asymmetry of the information. This implies that if $x$ is sufficiently high $(x>\bar{x})$, by altering only the ignorance contract the principal would be giving incentives to the informed party to sign the contract of the bad firm (i.e. the value of $e^{I}$ would fall below $\underline{e}$ ). The regulator avoids this by collapsing both contracts into a single one. ${ }^{8}$ As $x$ tends to 1 , the optimal contract converges to the one under adverse selection. The remaining characteristics of the contract are standard. There is non distortion at the top and only the informed good firm gets extra rents. The shape of the contract is presented in Figure 1.

[Insert Figure 1]

\section{Regulation under Partial Ignorance and Informed Third Par- ties}

In this section we study the role of third parties who may own some relevant information on the state of nature. We assume that the third parties may get to know whether the project to be implemented is highly damaging for the environment or not, by performing or buying studies of environmental impact.

We will consider two possible kinds of third parties. First, the environmentalists, defined as a pressure group only concerned about the environmental quality. This determines their behavior: if they get the information about the nature of the project, the environmentalists will only disclose it (make it public) if by doing it, they induce a lower level of pollution. In order to avoid problems of false claimings, we assume that the information the environmentalists get is hard evidence. Their degree of freedom is whether to disclose it or hide it, but they cannot falsely claim the project is of a certain type.

The second type of third-parties is given by the "independent" experts. When required, these agents perform tests and always disclose their findings to the principal. Hence, in terms

\footnotetext{
${ }^{7}$ At this point, one may think that the "quality" of the project generates a similar trade-off for the regulator as the presence or absence of ignorance. If the regulator had $\bar{\alpha}$ (the efficiency of the good project) as a choice variable, he would have to take into account that even if a better project generates less environmental damage, it is also more costly, since it allows the firm to ensure higher informational rents. It can be shown that in this model, the environmental effect always dominates the informational one. Therefore, it is never optimal for the regulator to select a highly damaging project.

${ }^{8}$ This feature of partial pooling when the information in the economy is sufficiently high is also present in Kessler (1998).
} 
of the information they provide, experts are superior to environmentalists. However, while the environmentalists typically privately pay for the information gathering costs, experts have to be compensated for the searching costs in which they incur. ${ }^{9}$

We will consider that the information is costly for the firm and for the third-parties. As it is also the case for the firm, the third parties's only decision is whether to buy this signal or not. The cost of the information is increasing on the accuracy of the test. We denote by $C_{x}$ the cost for the firm, and by $C_{z}$ that for the third parties. We assume that the costs are sufficiently low to ensure that, in any situation in which the agents have a potential gain from information, it is worth buying it. We are assuming that the environmentalists and the experts have access to the same information $(z)$ and do it at the same cost $\left(C_{z}\right)$. Since the acquisition of the information is unverifiable for the regulator, its costs are not included in the transfers of the contract. We assume that an expert has to be compensated for the costs of acquiring the information and that this is not the case for an environmentalist. Note that we consider that environmentalists privately pay for the costs of searching because they have stakes in regulation. This assumption can be relaxed, to encompass the case where the environmentalists' expenditures are partially compensated, provided this coverage is not complete ${ }^{10}$.

We treat both parties information acquisition analogously as the one of the firm. They obtain a signal $\left(s^{E}\right)$ defined as follows:

$$
\begin{aligned}
& s^{E}=\{\alpha\} \text { with probability } z \\
& s^{E}=\{\varnothing\} \text { with probability } 1-z .
\end{aligned}
$$

As before, the accuracy of the test (the value of $z$ ) is given; eventually the agents can only decide whether to perform it (alternatively, to buy it) or not.

An important point in our game concerns the timing at which the third party is called to participate. We consider two timings. In the first one the third party is asked to disclose its information at the beginning of the regulatory process. In the second one, it is only consulted after the firm has done its announcement. We will refer to these alternative temporal structures as "third-parties filter" and "third-parties check", respectively. Note that the role of the third party changes from one timing to the other. If the third party plays before (acting as a filter) it is providing information to the principal concerning the type of firm he is contracting with; if it plays after (as a check) it is also monitoring whether the firm was truthful when selfselecting. The timings of both configurations are summarized in what follows.

- "Third-parties filter" timing:

1. The third-party decides whether to buy or not the signal $s^{E}$.

\footnotetext{
${ }^{9}$ The third parties' behavior can be seen from an "advocacy" perspective, considering that in fact their duty is to search for evidence pro or against the project being highly polluting. In the terminology of Dewatripont and Tirole (1999), the environmentalists would be close to the "advocates" who only care about the information supporting one side, while the experts would be similar to the "non-partisans".

${ }^{10}$ The interpretation for this assumption is that even if an organization like Greenpeace has access to public funds, its actions are mainly financed by the contributions of its members worldwide.
} 
2. The principal asks the third-party to voluntarily disclose what he knows.

3. If the firm's project was not revealed by the third-party, the firm can learn (buy) the signal $s^{F}$.

4. The contract is designed.

5. If the quality of the project was revealed, the firm is given the corresponding optimal contract (without rents). Otherwise, the firm uses its information to selfselect.

6. The project is undertaken.

Under this timing, we make use first of the information of the informed agents. If they fail to uncover the type of project, the firm can have incentives to privately gather information. The other possibility is:

- "Third-parties check" timing:

1. The firm learns (buys) $s^{F}$.

2. The contract is designed.

3. The firm uses its information to selfselect.

4. The third-party decides whether to buy or not the signal $s^{E}$.

5. If the contract signed is not the efficient firm's one, we ask the third-party to disclose what he knows about the project's efficiency.

6. It the firm is caught lying it is punished, and its contract reassigned.

7. The project is undertaken.

In this alternative temporal structure, the informed agents' knowledge is used to threaten the "good" firm and prevent it from signing other type's contract. The third parties also help to place correctly those firms who remained ignorant after selfscreening.

\subsection{Environmentalists}

Environmentalists are only concerned by the environmental damage. If they know that the firm's project is efficient, the environmentalists will be interested in disclosing it (revealing to the regulator that the firm is efficient). The reason is that an efficient firm is asked to exert a higher effort than a firm who is ignorant about its type. Then, in this case, the incentives of the pressure group and the principal are aligned. On the contrary, when the environmentalists learn that the firm's project is inefficient, they have incentives to keep this information for themselves, since the effort asked to the ignorant firm is always higher or equal than the one of the inefficient firm. This is so under both timings. Therefore, the environmentalists report $\left(R^{E}\right)$ is:

$$
R^{E}=\left\{\begin{array}{c}
\bar{\alpha} \text { if } s^{E}=\{\bar{\alpha}\} \\
\emptyset \text { if } s^{E} \in\{1, \emptyset\}
\end{array} .\right.
$$


After the report of the environmentalists, the regulator updates the probabilities. If the report is $R^{E}=\bar{\alpha}$, the posterior is that the firm is efficient; if the report is $R^{E}=\emptyset$ and given the environmentalists behavior, the updated probability that the firm is efficient $(\widetilde{v})$ is smaller:

$$
\widetilde{v}=\operatorname{Pr}\left(\alpha=\bar{\alpha} \mid R^{E}=\{\emptyset\}\right)=\frac{(1-z) v}{(1-z v)}<v .
$$

Let us denote the social welfare (gross of firm's rents) by $\bar{W}$ if the project is good, by $\underline{W}$ if it is bad, and by $\widetilde{W}(\widehat{\alpha}(v))$ if the regulator is ignorant about its type:

$$
\begin{aligned}
\bar{W} & =S-V(\bar{\alpha}, \bar{e})-(1+\lambda) \Psi(\bar{e}) \\
\underline{W} & =S-V(1, \underline{e})-(1+\lambda) \Psi(\underline{e}) \\
\widetilde{W}(\widehat{\alpha}(v)) & =S-V\left(\widehat{\alpha}(v), e^{I}\right)-(1+\lambda) \Psi\left(e^{I}\right) .
\end{aligned}
$$

When the environmentalists are used as a filter, the objective function of the regulator is:

$$
\begin{aligned}
& W_{e n-f}=z v \bar{W}+(1-z v) x\left[\widetilde{v}\left(\bar{W}-\lambda \bar{U}_{e n-f}\right)+(1-\widetilde{v})\left(\underline{W}-\lambda \underline{U}_{e n-f}\right)\right] \\
& +(1-z v)(1-x)\left(\widetilde{W}(\widehat{\alpha}(\widetilde{v}))-\lambda U_{e n-f}^{I}\right)-C_{z}-(1-z v) C_{x} .
\end{aligned}
$$

Here, as the environmentalists are the first to move, the probabilities are already updated when the firm is asked to selfselect. Moreover, the environmentalists always incur in costly searching while the firm only does it if its type was not revealed by the environmentalists.

When they are used as a check:

$$
\begin{aligned}
& W_{e n-c}=x\left[v\left(\bar{W}-\lambda \bar{U}_{e n-c}\right)+(1-v)\left(\underline{W}-\lambda \underline{U}_{e n-c}\right)\right]+ \\
& (1-x)\left[z v \bar{W}+(1-z v)\left(\widetilde{W}(\widehat{\alpha}(\widetilde{v}))-\lambda U_{e n-c}^{I}\right)\right]-C_{x}-(1-x v) C_{z} .
\end{aligned}
$$

In this case, the Bayesian updating only affects the expected efficiency parameter in the ignorance contract $(\widehat{\alpha}(\widetilde{v}))$. In this configuration it is the firm the one that always acquires information, while the environmentalists only do it with a certain probability.

As it will be shown later, the average costs of searching will be crucial for the selection between the alternative configurations. Therefore, hereinafter we denote $\frac{C_{z}}{z}=A C_{z}$ and $\frac{C_{x}}{x}=$ $A C_{x}$. By confronting the two previously explained timings, we get the following Proposition:

Proposition 1 For a given $(x, z)$, in the presence of imperfectly informed environmentalists:

i.- The optimal contract for the firm under the two timings is the same.

ii.- The timing with environmentalists filtering dominates the one with environmentalists checking if and only if $A C_{z}<A C_{x}$.

The first part of Proposition 1 makes reference to the allocative efficiency attained under the two alternative timings. We prove that the objective function of the regulator only differs in the associated costs of gathering information, and that therefore, the optimal contract for the firm is identical. The intuition is related to the expected gains under both timings. Using the environmentalists as a filter, the regulator only pays the informational rents with a certain probability. On the contrary, using them as a check does not reduce the likelihood of paying the 
rents, but reduces the quantity. The reason is that the deviation possibilities are less attractive having the environmentalists as "watchdogs". We prove that these two effects are quantitatively equivalent in expected terms, hence the optimal contracts are identical. ${ }^{11}$ Thus, the key point is the cost of acquiring the information. The second part of the Proposition states that this cost comparison determines the best regulatory timing by comparing the average costs of both tests. This relates to the fact that average costs indicate the efficiency of the test, as they provide a measure of the costs rescaled by the probability that the expenditure results in a real knowledge of the type of project. As we have shown that the timing has no impact on the contract, the regulator prefers to place the environmentalists as a filter only if their test has lower average costs than that of the firm. Otherwise, the principal prefers to let the firm selfscreen first and use the presence of environmentalists only as a threat.

If the environmentalists privately choose the timing, or if the principal cannot force them to act when he wants to, then the environmentalists always prefer the "checking" timing. The reason is that they induce the same contract as in the "filtering" one, but incur in costly searching less often. However, the incentives to act afterwards would create a problem of credibility for the environmentalists, because the firm will behave as predicted by the contract, only if it is sure that the environmentalists will ex-post search. This problem is not present if it is the regulator, who decides when to ask for the third-party's report.

\subsection{Independent Experts}

We move now to a situation in which the regulator can hire a group of experts who have no personal interest in the regulatory process, and simply perform the task they have been asked to. If an expert is contracted, he performs the tests and always truthfully reports his findings. This is equivalent to having a regulatory agency whose duty is to try to screen the firm and give the principal the information he needs for regulating the firm. ${ }^{12}$ Since experts only perform those tests because we ask them to, they have to receive, at least, the corresponding costs. Thus, by using experts, the regulator publicly finances the projects' screening and incurs in the extra costs $\lambda$.

Finally, note that as the experts' reporting policy is independent from what they learn (always report truthfully), if the experts claim that they did not learn the type of project, the posterior probability that the firm is good will remain unaltered. The objective function when the experts are used as a filter is:

$$
\begin{aligned}
& W_{e x-f}=z[v \bar{W}+(1-v) \underline{W}]+(1-z)(1-x)\left(\widetilde{W}(\widehat{\alpha}(v))-\lambda U_{e x-f}^{I}\right) \\
& +(1-z) x\left[v\left(\bar{W}-\lambda \bar{U}_{e x-f}\right)+(1-v)\left(\underline{W}-\lambda \underline{U}_{e x-f}\right)\right]-(1+\lambda) C_{z}-(1-z) C_{x} .
\end{aligned}
$$

\footnotetext{
${ }^{11}$ This result is only true under the assumption that the principal is risk neutral. If he is risk averse, the timing with environmentalists as a check strictly dominates the one with environmentalists as a filter (disregarding the search costs).

${ }^{12}$ Laffont and Tirole (1991) construct a model of "Regulatory Capture", in which the principal asks a regulatory agency to screen the firms. However their analysis is completely different from ours since they are interested in contracts that prevempt the firm from bribing the regulatory agency.
} 
The one with the experts checking is:

$$
\begin{aligned}
& W_{e x_{-} c}=x\left[v\left(\bar{W}-\lambda \bar{U}_{e x-c}\right)+(1-v)\left(\underline{W}-\lambda \underline{U}_{e x-c}\right)\right]+(1-x) z[v \bar{W}+(1-v) \underline{W}] \\
& (1-x)(1-z)\left(\widetilde{W}(\widehat{\alpha}(v))-\lambda U_{e x-c}^{I}\right)-C_{x}-(1-x v)(1+\lambda) C_{z} .
\end{aligned}
$$

Comparing both timings, we have the following proposition:

Proposition 2 For a given $(x, z)$, in the presence of imperfectly informed experts:

i.- The optimal contract under the two timings is the same.

ii.- The timing with experts filtering dominates the one with experts checking if and only if $v(1+\lambda) A C_{z} \leq A C_{x}$.

The first part of Proposition 2 is analogous to the one of the previous subsection. On the contrary, the second part presents some new features that are worth explaining. Even if the important measure continues to be the average costs, new effects arise that influence the decision. First, with "experts filter" we always incur in the extra costs $\lambda$ while with the other timing, this only happens with a certain probability. Thus, "experts filter" only dominates provided the extra cost of public funds $(\lambda)$ is not too high. The second effect is that while all the informative reports $\left(R^{E} \neq \emptyset\right)$ of the experts when they are used as a filter are useful, when they check some are ex-post useless. The reason is that in order to keep a credible threat over the firms, with the "experts check" the regulator has to double screen not only those who reported to be ignorant about their type, but also the ones who claimed to be inefficient. Therefore, the experts perform checks that in equilibrium are useless (by the revelation principle no firm lies), but that are needed ex-ante, precisely to sustain the equilibrium. This second effect favors the optimality of the timing with "experts filter".

\subsection{Experts versus Environmentalists}

Now we present a comparison between the situation with experts and with environmentalists. Experts are more costly, but they are also more efficient because they never hide information to the principal.

First let us concentrate on the expected benefits of the different alternatives ignoring by now the cost of the signal. From our assumption on the behavior of the different third parties, it is obvious that the use of experts can never be inferior in terms of expected revenues than the use of environmentalists. Proposition 3 states when this advantage is strictly positive.

Proposition 3 If $C_{z}=C_{x}=0$, there exists a $\bar{x}<1$, such that:

i.- $\forall x<\bar{x}$, the optimal contract in the presence of experts dominates the one with environmentalists.

ii.- $\forall x \geq \bar{x}$, the optimal contract in the presence of experts and in the presence of environmentalists are equally efficient. 
Proposition 3 tells us that even if the experts always provide the principal with more information in expected terms, this information not always leads to a more efficient contract. If the test the firm has access to is sufficiently informative (and therefore the probability that a firm remains ignorant about its type after trying the test is low enough), this information surplus is completely useless. The threshold $\bar{x}$ corresponds to the level obtained in Lemma 1 , from which on the resulting contract is pooling for the inefficient and the ignorant firms. Even if the environmentalists label as ignorant firms they know that are inefficient, and that it is not the case with experts, in this region this has no effect on the contract because both types of firms are given the same incentive scheme. This result is interesting because it shows how relying on interested parties' information can be optimal, even if the agents do not have incentives to reveal everything they know. It all depends on whether the principal needs all the information, or as in this case it requires only the part that is revealed. Obviously if the value of $x$ is below that threshold, the extra information the experts give is relevant for the principal and therefore leads to a better contract.

We consider now the revenues net of information searching costs, and compare the two alternative third-parties, and the different timings.

Let us define a threshold for the shadow cost of public funds

$$
\bar{\lambda} \equiv x \frac{A C_{x}-v \max \left\{A C_{x}, A C_{z}\right\}}{A C_{z}} .
$$

From the construction of $\bar{\lambda}$ it can be seen that if $A C_{x}>A C_{z}$ then $\bar{\lambda}>0$, but if $A C_{x}<A C_{z}$ it can be the case that $\bar{\lambda}<0$.

Proposition 4 If $C_{x}>0$ and $C_{z}>0$ then the best regulatory scenario is:

1. If $x \geq \bar{x}$

$$
\begin{array}{cc}
\text { experts filter } & \text { if } \lambda \leq \bar{\lambda} \\
\text { environmentalists filter } & \text { if } \lambda>\bar{\lambda} \text { and } A C_{z} \leq A C_{x} \\
\text { environmentalists check } & \text { if } \lambda>\bar{\lambda} \text { and } A C_{z}>A C_{x} .
\end{array}
$$

2. If $x<\bar{x}$, there exists a $\overline{\bar{\lambda}}>\bar{\lambda}$ such that the optimal configuration is the same as above, replacing $\bar{\lambda}$ by $\overline{\bar{\lambda}}$.

Proposition 4 shows how for low values of $\lambda$, the optimal regulatory structure is the one with "experts filter". The reason is that when the cost of public funds is small, then the structure with experts dominates and, as we already highlighted, using the experts as a check entails a relative disadvantage due to ex-post unnecessary double-checkings. When $\lambda$ is sufficiently high the regulator is better off by using environmentalists and their optimal position in the timing is determined by the comparison of the average costs.

It is also worth noting how the threshold $\bar{x}$ determines two regions, that differ in the amount of extra cost $(\lambda)$ that the regulator is willing to bear in order to have an unbiased expert in 
the regulatory process. When $x \geq \bar{x}$, we already know by Proposition 3 that the nature of the third-party makes no difference in the resulting contract. Hence, the choice is made only by comparing the costs of gathering information with experts, with those in the configuration with environmentalists. On the contrary, when $x$ falls below the threshold $\bar{x}$, the optimal contracts do not exhibit the feature of partial pooling and hence, by using experts, the regulator acquires more information that induces a more efficient contract. Therefore, in this region the decision is not only based in the costs but also in the higher efficiency attained with the experts. We show that this efficiency gain decreases as $\lambda$ increases and this feature, together with the cost comparison, allows us to ensure that there exists a new threshold for the cost of raising public funds, higher than $\bar{\lambda}$. From this level $\overline{\bar{\lambda}}$ on, it is better for the regulator to deal with informed environmentalists instead of experts.

\section{Endogeneizing the Ignorance}

In this Section we go one step forward and consider the possibility that the firm decides the amount of ignorance it wants to suffer, which is given by the accuracy of the test it buys. We compare the choice of the firm with the one the regulator would have made. We also provide some insights on how the third-parties' timing selection problem is affected when the level of ignorance is endogenous.

As we argued in the introduction, due to the nature of our problem, it is reasonable to consider that the firm's decision is not perceived by the regulator when designing the contract. Hence we will develop the analysis in a simultaneous setting between the firm (choosing the value of $x$ ) and the regulator (designing the contract).

\subsection{Firm's Choice of ignorance}

In this Subsection we will not consider the effect of the presence of third-parties. The costs of the test are given by $C(x)$, with $C^{\prime}(\cdot) \geq 0, C^{\prime \prime}(\cdot)>0$. In order to ensure an interior solution for the firm's problem, we will assume that $C(0)=C^{\prime}(0)=0$, and $\lim _{x \rightarrow 1} C^{\prime}(x)=+\infty$. Therefore the expected profits of a firm that buys an "accuracy" $x$ for the signal $s^{F}$ are:

$$
E \Pi(x)=x v \Phi\left(\max \left(e^{I}, \underline{e}\right)\right)-C(x) .
$$

That is, the firm always incurs in the cost of searching, and only gains profits (informational rents) if it learns that the project it is endowed with is efficient. In order to be able to derive solutions for the game, we will consider that cost of the pollution abatement effort is $\Psi(e)=\frac{e^{2}}{2}$. We also assume that ex-ante the two projects are equally likely $\left(v=\frac{1}{2}\right)$.

We begin by considering the case in which $C(x)=0 \forall x$, this will help us to understand the principal's behavior toward the presence of ignorance. Let us denote by $x^{R}$ the optimal regulator's choice of $x$, i.e. the level of firm's information that maximizes social welfare.

Let us define a threshold for $\lambda, \lambda(\bar{\alpha})=\frac{(\bar{\alpha}-1) \bar{\alpha}^{2}}{1+3 \bar{\alpha}+2 \bar{\alpha}^{2}-2 \bar{\alpha}^{3}}, \lambda(\bar{\alpha})$ is such that $\lambda(1)=0, \lambda^{\prime}(\bar{\alpha})>0$, and there exists a $\bar{\alpha}_{\max }$ such that $\lim _{\bar{\alpha} \rightarrow \bar{\alpha}_{\max }} \lambda(\bar{\alpha})=+\infty$. 
Proposition 5 If $C(x)=0$, then the regulator's choice of $x\left(x^{R}\right)$ is:

i. $-\forall \bar{\alpha} \leq \bar{\alpha}_{\max }$,

$$
\begin{aligned}
& \text { If } \lambda \leq \lambda(\bar{\alpha}) \quad \text { then } x^{R}=1 \text { (adverse selection) } \\
& \text { If } \lambda>\lambda(\bar{\alpha}) \quad \text { then } x^{R}=0 \text { (full ignorance) }
\end{aligned}
$$

ii. $-\bar{\alpha}>\bar{\alpha}_{\max }$, then $x^{R}=1$ (adverse selection)

Proposition 5 brings to place the insight given after Lemma 1 about the conflicting effects that the presence of ignorance had for the efficiency of the contract. Here we show that the regulator's objective function in the absence of costs of gathering information is convex with respect to the information of the firm. Even if more information is costly in terms of the rents he has to pay, the marginal effect is decreasing because as $x$ increases, the optimal contract becomes more distorted (precisely to decrease the informational rents). The highest allocative efficiency is attained when $x=1$, because there is a perfect assignment of the types to their corresponding levels of effort. However, this is costly in terms of informational rents paid. Therefore this will only be the solution provided the extra costs of public funds are not too high.

For values beyond that threshold, it is worth for the regulator to sacrifice completely allocative efficiency to avoid paying costly rents and hence $x=0$. The value of $\bar{\alpha}$ affects this decision because it reflects the technological advantage of the good project. The higher the level of $\bar{\alpha}$, the more efficient is the good project in reducing the environmental damage, and hence the more the regulator will be sacrificing by choosing $x=0$. Thus, the regulator will be willing to incur in higher costs $(\lambda)$ in order to contract an informed firm. The Proposition shows how if the value of $\bar{\alpha}$ is sufficiently high (beyond a certain threshold $\bar{\alpha}_{\max }$ ), the principal wants to deal with a perfectly informed agent no matter the value of $\lambda$. However this region is very small, $\bar{\alpha}_{\max }$ is very close to the upper bound of the domain of $\bar{\alpha} .{ }^{13}$

In the following proposition we will make explicit the relation between the firm's choice of $x$, and what the principal would choose, i.e. the amount of information that the regulator would like the firm to have in the presence of costs of gathering information $(C(x)>0)$. We will restrict our analysis to the range of parameter values for which both problems yield interior solutions. ${ }^{14}$ Let us denote by $x^{F}$ the level of information acquisition chosen by the firm.

Proposition 6 When acquiring information is costly, $\exists \widetilde{x}(\beta, \lambda)<1$, s.t.:

$$
\begin{aligned}
& \text { If } x^{F}<\widetilde{x}(\beta, \lambda) \text {, then } x^{F}>x^{R} \text {. } \\
& \text { If } x^{F}=\widetilde{x}(\beta, \lambda) \text {, then } x^{F}=x^{R} \text {. } \\
& \text { If } x^{F}>\widetilde{x}(\beta, \lambda) \text {, then } x^{F}<x^{R} \text {. }
\end{aligned}
$$

Proposition 6 has the following interpretation. For each combination of the parameters there exists a threshold in the level of ignorance $(\widetilde{x}(\beta, \lambda))$ such that, if the firm chooses a value of $x$

\footnotetext{
${ }^{13} \bar{\alpha}_{\max }=1.918$ and recall that the domain of $\bar{\alpha}$ is $\bar{\alpha} \in(1,2]$.

${ }^{14}$ It is straightforward from our construction that the firm's program always has a unique interior solution. In the proof of Proposition 6 we provide a sufficient condition for this to happen in the program of the principal. The condition requires that some information is always profitable.
} 
below this value (chooses a relatively high degree of ignorance), then the regulator's choice of $x$ would be even lower. On the contrary, if the firm's choice exceeds the critical value $\widetilde{x}(\beta, \lambda)$, then the regulator would like to face an even better informed firm. Therefore, the firm's decision is generically non-optimal, and always moderate with respect to the socially optimal level of information. The reason for this is the opposite behavior of the marginal benefits from an increase in $x$, for the firm and the regulator. For the regulator, a higher level of $x$ generates not only a better allocation of efforts but also a decrease in the amount of rents to be paid (the distortion in the contract is increasing in $x$ ). On the contrary, for the firm an increase of $x$ decreases the marginal benefits as it makes the contract tougher. This yields a more extreme behavior for the regulator concerning the firm's acquisition of information.

At this point, we can analyze the impact of the cost of public funds on the regulator's choice of $x$. An increase in $\lambda$ has a direct negative effect over the incentives of the principal to deal with an informed firm: the higher is $x$, the more likely it is that the firm gets extra rents, and these rents are more costly the higher is the value of $\lambda$. However, this is corrected by the contract because when the cost increases, the contract is distorted more in order to pay less rents. The following proposition gives the outcome to the interaction of these contrary effects.

Proposition 7 An increase in $\lambda$ decreases the socially optimal level of information acquisition:

$$
\frac{\partial x^{R}}{\partial \lambda}<0
$$

Proposition 7 shows that the direct negative effect always dominates the positive one. That is, even if the regulator reacts to an increase of $\lambda$ with a more demanding contract, the overall effect makes less attractive dealing with an informed firm.

One last comment is that we have not found an strategic value for ignorance, as Kessler (1998) did. The difference with her analysis is that she considered that the firm's choice of ignorance was prior to the design of the contract, and that therefore, the firm internalized the effect of its choice on the contract. Hence, the firm strategically chose to bear a certain degree of ignorance in order to reduce the toughness of the incentive contract designed by the principal. As we have already argued, we consider that due to the nature of environmental regulation problems, it is more reasonable to perform the analysis in a simultaneous setting, where no such strategic considerations are present. Therefore, our analysis relies on a different interaction of effects. What determines our results is the difference in the value the players give to an increase in the amount of information available for the firm.

\subsection{On the Location of the Third-Parties with Endogenous Ignorance}

The fact that the firm's decision is almost never aligned with the regulator's gives a new dimension to the problem with informed third parties. The choice of the optimal timing has to take into account the different incentives to search that they give to the firm.

In this subsection we investigate this issue. Due to the impossibility to fully characterize the results, we only analyze the effects that appear in the selection of the best type of third- 
party, and on its optimal location. We start our analysis by identifying the different levels of information acquisition by the firm that the alternative timings induce.

Lemma 2 The firm's choice of information under the alternative timings is:

$$
x_{e x-f}^{F}>x_{e n-f}^{F}>x_{e n-c}^{F}=x_{e x-c .}^{F} .
$$

Lemma 2 shows that the firm's incentives to gather information are higher when the third parties are used as a filter, than when they are act as a check. Moreover, we find that the highest incentives for information acquisition are given by the experts, when they are placed at the beginning of the regulatory process filtering the firms.

With this result in mind, we can now state the main effects that determine which is the optimal regulatory timing when ignorance is endogenous. These effects are:

1. When the level of ignorance is exogenous, the best regulatory structure is "experts filter" for low values of $\lambda$ (Proposition 3).

2. The lower the costs of public funds $(\lambda)$, the higher the amount of information that the regulator wants the firm to have (Proposition 7).

3. The configuration leading to the highest information acquisition by the firm is "experts filter" (Lemma 2).

Therefore, when the extra costs of public funds are small, all the effects point toward the same timing as the optimal one: experts filter. For high cost levels, we showed that environmentalists dominate when the ignorance is exogenous, and it is more likely that the regulator is interested in restricting the firm's choice of information, since it is more costly. Hence, when the extra costs of public funds are important, the best regulatory structure is one with environmentalists, with its location on the timing being determined by the comparison of the average costs.

\section{Conclusion and Policy Implications}

The objective of this article has been two fold. On the one hand, we have studied the impact of the presence of ignorance on the environmental regulatory performance, first considering it exogenous, and afterwards giving the firm the capacity to choose the degree of ignorance it wants to suffer. On the other hand, we also analyzed the issue of having other agents (third-parties different from the firm and the principal), who posses, or may gather, some relevant information. We addressed the question of the extent to which the nature of the informed agent, an interested party (environmentalists) or a neutral one (unbiased experts), and his location in the timing, affected the regulatory process. The study is developed in a model of regulation of a firm that has been entitled to implement an environmentally damaging project. 
We have chosen to consider that the source of the informational problem is extrinsic to the firm. Contrary to other papers in the literature, in which what the principal did not know was the cost of the pollution abatement effort, we have assumed that the informational asymmetry concerns the impact of the project on the environment. We argue that this construction makes more credible the presence of ignorance and of potentially informed third parties that can perform, for instance, studies of environmental impact.

We think that some of the results obtained in our analysis have implications that are worth noting. Specifically we will focus on two aspects: the choice of the best regulatory structure and the consequences of relying on the information provided by interested parties (in our case the environmentalists).

\subsection{The Choice of the Optimal Regulatory Structure}

The choice of the location of third parties in the regulatory timing can be interpreted as the determination of who should take the initiative to screen the project and evaluate its environmental effects. The "Third-Parties filter" structure corresponds to a situation in which it is the public authority who takes this initiative, by asking the agents with technical capacity to perform the study (the experts or the environmentalists) to do it, leaving the firm's selfscreening as a secondary option. Conversely, in the "Third-Parties check" timing the regulator lets the firm move first, and only uses its capacity to ask for a study as a threat to correct the strategic behavior of the firm.

If we study the actual environmental legislation in force in the EU, we find that the Directive $85 / 337 / E E C$, and its amendment Directive $97 / 11 / E C$, legislate in favor of the first timing as they say "Member States shall adopt all measures necessary to ensure that, before consent is given, projects likely to have significant effects on the environment by virtue inter alia, of their nature, size or location are made subject to an assessment with regard to their effects".

Our results support this structure as optimal, provided the extra costs that the public sector performance entails are not too high. Moreover, we find another interesting effect of this timing. We show that this structure with an ex-ante publicly induced study, not only does not substitute the firm's information acquisition, but also induces a higher effort by the firm to become informed about the characteristics of the project it is endowed with.

Hence, these Directives seem to be well designed to protect the environment, as on top of their direct effect (the assessment of the effects of the project), they add an indirect effect of inducing the firm that will implement the project to invest more in knowing its characteristics, thus yielding a better allocation of efforts (the actual process of construction) to the environmental characteristics of the project. However, one should note that the welfare maximizing option for the regulator can be to avoid paying rents at all cost. This is the case if the inefficiency that collecting fund causes $(\lambda)$ is very high, as it is in developing countries. We show that, in this case, the regulator would like to deal with a "poorly-informed" firm, and hence he should place the checks ex-post. 


\subsection{The Role of the Environmentalists as Information Providers}

Our work has also some implications concerning the effects of relying on interested parties' information. In our framework, the environmentalists' information is "hard evidence"; this assumption is introduced to keep the model tractable and avoid situations in which both, the environmentalists and the firm, lie and blame each other for misreporting, with a principal unable to know who actually lied. This construction limits the strategic behavior of the environmentalists as it precludes them from, for instance, exaggerating their evidence, in order to induce a more severe regulatory action. In spite of that, this simple construction still leaves room for the interested party to affect, through their "evidence disclosure" policy, the amount of information the principal posses when designing the policy.

We show that, in our context, the information disclosed by the environmentalists may be sufficient for the principal to take his decision, due to the fact that the presence of ignorance generates an equal treatment of different types (a partial pooling feature) in the optimal incentive contract. Hence, our model can be taken as a starting point for the analysis of the circumstances under which the regulatory process may benefit from the information provided by interested parties. This study should be based on the identification of the relative strengths of several opposing forces. On the one hand, the lower cost (as it is in our model) and possibly the higher accuracy (precision) of their information. And on the other, the bias that their selfish motivation will place on their information disclosure. This kind of studies can be very relevant for the design of future policy measures.

\section{Appendix.}

\section{Proof of Lemma 1:}

If $s^{F}=\{\varnothing\}$ or $s^{F}=\{1\}$ the participation constraint of the firm is binding:

$$
\begin{aligned}
U^{I} & =t^{I}-\Psi\left(e^{I}\right)=0 \Rightarrow t^{I}=\Psi\left(e^{I}\right) \\
\underline{U} & =\underline{t}-\Psi(\underline{e})=0 \Rightarrow \underline{t}=\Psi(\underline{e}) .
\end{aligned}
$$

If $s^{F}=\{\bar{\alpha}\}$, the firm can pretend to be inefficient and reduce its effort. Denoting by $\gamma$ the reduction that the firm can make in its effort without altering the final level of environmental damage: $V(1, \underline{e})=V(\bar{\alpha}, \underline{e}-\gamma) \Rightarrow D-\underline{e}=D-\bar{\alpha}(\underline{e}-\gamma) \Rightarrow \gamma=\frac{\bar{\alpha}-1}{\bar{\alpha}} \underline{e}$. With this, the incentive compatibility constraint of the efficient firm with respect to the inefficient firm: $\bar{U}=\bar{t}-\Psi(\bar{e}) \geq$ $\underline{t}-\Psi(\underline{e}-\gamma(\underline{e}))=\Psi(\underline{e})-\Psi(\underline{e}-\gamma(\underline{e})) \equiv \Phi(\underline{e})>0 \Longrightarrow \bar{U} \geq \Phi(\underline{e})$.

Analogously, for the ignorant firm: $\bar{U} \geq \Phi\left(e^{I}\right)$.

Therefore, $\bar{U}=\max \left[\Phi(\underline{e}), \Phi\left(e^{I}\right)\right]$, as $\Phi^{\prime}(e)>0$, the condition is equivalent to $\bar{U}=\Phi\left(\max \left[\underline{e}, e^{I}\right]\right)$.

The objective function of the regulator under ignorance $\left(W^{I}\right)$ is the following:

$$
\begin{aligned}
& \max _{e^{I}, \bar{e}, \underline{e}} W=(1-x)\left(S-V\left(\widehat{\alpha}, e^{I}\right)-(1+\lambda) \Psi\left(e^{I}\right)\right)+ \\
& \quad x\left[v\left(S-V(\bar{\alpha}, \bar{e})-(1+\lambda) \Psi(\bar{e})-\lambda \Phi\left(\max \left[\underline{e}, e^{I}\right]\right)\right)+(1-v)(S-V(1, \underline{e})-(1+\lambda) \Psi(\underline{e}))\right] .
\end{aligned}
$$


In the undistorted contract, $e^{I}>\underline{e}$, hence we start by computing the contract for the case $\max \left[\underline{e}, e^{I}\right]=e^{I}$. The resulting optimal contract is:

$$
\begin{aligned}
\bar{e} \text { is s.t. } \Psi^{\prime}(e) & =\frac{\bar{\alpha}}{1+\lambda} \\
\underline{e} \text { is s.t. } \Psi^{\prime}(e) & =\frac{1}{1+\lambda} \\
e^{I} \text { is s.t. } \Psi^{\prime}(e) & =\frac{\widehat{\alpha}}{1+\lambda}-\frac{x v}{1-x} \frac{\lambda}{1+\lambda} \Phi^{\prime}(e) .
\end{aligned}
$$

This is the case provided $\underline{e} \leq e^{I}$, which holds if $x \leq \bar{x}<1$, with $\bar{x}=\frac{\bar{\alpha}-1}{\bar{\alpha}-1+\lambda \Phi^{\prime}(e)}$.

If not, then $\max \left[\underline{e}, e^{I}\right]=\underline{e}=e^{I}$. The contract is:

$$
\begin{aligned}
\bar{e} \text { is s.t. } \Psi^{\prime}(e) & =\frac{\bar{\alpha}}{1+\lambda} \\
e^{I} \text { and } \underline{e} \text { are s.t. } \Psi^{\prime}(e) & =\frac{1}{1+\lambda}\left(\frac{(1-v) x+\widehat{\alpha}(1-x)}{1-x v}\right)-\frac{x v}{1-x v} \frac{\lambda}{1+\lambda} \Phi^{\prime}(e) .
\end{aligned}
$$

This holds if $x>\bar{x}$. The second order conditions are fulfilled.

\section{Proof of Proposition 1:}

1) As $z$ and $x$ are given constants, to check if the optimal contracts are the same we do not have to care about the searching costs.

When comparing the objective function, we have to take into account that:

$$
\begin{aligned}
& \bar{U}_{e n-f}=\Phi\left(\max \left(e^{I}, \underline{e}\right)\right) \\
& \bar{U}_{e n-c}=(1-z) \Phi\left(\max \left(e^{I}, \underline{e}\right)\right) \\
& \underline{U}_{e n-c}=\underline{U}_{e n-f}=U_{e n-c}^{I}=U_{e n-f}^{I}=0 .
\end{aligned}
$$

Algebraic manipulations show that $W_{e n-f}^{I}-W_{e n-c}^{I}=v\left(z C_{x}-x C_{z}\right)$, hence the resulting levels of $\left(\bar{e}, \underline{e}, e^{I}\right)$ and of $\left(\bar{t}, \underline{t}, t^{I}\right)$ are the same under both timings.

2) Using the results of 1 ), we can write:

$$
W_{e n-f}^{I}-W_{e n-c}^{I}=v z x\left(\frac{C_{x}}{x}-\frac{C_{z}}{z}\right) .
$$

Therefore, $W_{e n-f}^{I}-W_{e n-c}^{I}>(=) 0$ iff $\frac{C_{x}}{x}>(=) \frac{C_{z}}{z}$.

\section{Proof of Proposition 2:}

Completely analogous to the previous Proposition.

\section{Proof of Proposition 3:}

By Lemma 1, we know that $\exists \bar{x}<1$, such that $\forall x \geq \bar{x}, \underline{e}=e^{I}$. It can be shown that this threshold is the same in the programs with experts and with environmentalists.

$\forall x<\bar{x}$, the contract with experts trivially dominates the one with environmentalists, because it gives a lower fraction of the population, the pooling "ignorance" contract that is less efficient than the corresponding separating one. 
$\forall x \geq \bar{x}$, Algebraic manipulations show that the difference $W_{e x}^{I}\left(\bar{e}, \underline{e}=e^{I}\right)-W_{e n}^{I}\left(\bar{e}, \underline{e}=e^{I}\right)$, only consists of searching costs, and that hence, the two contracts are the same.

\section{Proof of Proposition 4:}

1) If $x \geq \bar{x}$, we know by Proposition 3 that the optimal decision only depends on the searching costs:

1.1.- If $\frac{C_{z}}{z}<\frac{C_{x}}{x}$, we already proved in Proposition 1 that $W_{e n-f}^{I}>W_{e n-c}^{I}$. It can be shown that $W_{e n-c}^{I}>W_{e x-f}^{I}$ iff $\lambda>x \frac{(1-v) \frac{C_{x}}{x}}{\frac{C_{z}}{z}}$.

Analogously, we find that $W_{e n-f}^{I^{z}}>W_{e x-c}^{I}, \forall \lambda>0$.

1.2.- If $\frac{C_{z}}{z}>\frac{C_{x}}{x}$, we already proved in Proposition 1 that $W_{e n-f}^{I}<W_{e n-c}^{I}$. It can be shown that $W_{e n-c}^{I}>W_{e x-f}^{I}$ iff $\lambda>\frac{\frac{C_{x}}{x}-v \frac{C_{z}}{z}}{\frac{C_{z}}{z}}$.

Analogously, we find that $\frac{\frac{c}{z}}{W_{e n-c}^{I}}>W_{e x-c}^{I}, \forall \lambda>0$.

Combining both regions we find that there exists a $\bar{\lambda} \equiv x \frac{A C_{x}-v \max \left\{A C_{x}, A C_{z}\right\}}{A C_{z}}$ such that,

$$
\begin{array}{cc}
\text { "experts filter" dominates } & \text { if } \lambda \leq \bar{\lambda} \\
\text { "environmentalists filter" dominates } & \text { if } \lambda>\bar{\lambda} \text { and } A C_{z} \leq A C_{x} \\
\text { "environmentalists check" dominates } & \text { if } \lambda>\bar{\lambda} \text { and } A C_{z}>A C_{x} .
\end{array}
$$

2) For $x<\bar{x}$, the cost comparison among the different specifications is not altered, but there is also a difference in the resulting contract, leading to a more efficient final allocation in the presence of experts. Proposition 3 ensures that the different location in the timing does not alter the resulting contract, the optimal efforts in the contract with experts $\left(e_{\exp }\right)$ and with environmentalists $\left(e_{\text {env }}\right)$ are:

$$
\begin{aligned}
\bar{e}_{e n v} & =\bar{e}_{\exp } \text { are s.t } \Psi^{\prime}(e)=\frac{\bar{\alpha}}{1+\lambda} \\
\underline{e}_{e n v} & =\underline{e}_{\exp } \text { are s.t } \Psi^{\prime}(e)=\frac{1}{1+\lambda} \\
e_{\text {exp }}^{I} \text { is s.t. } \Psi^{\prime}(e) & =\frac{\widehat{\alpha}(v)}{1+\lambda}-\frac{x v}{1-x} \frac{\lambda}{1+\lambda} \Phi^{\prime}(e) \\
e_{e n v}^{I} \text { is s.t. } \Psi^{\prime}(e) & =\frac{\widehat{\alpha}(\tilde{v})}{1+\lambda}-\frac{x \tilde{v}}{1-x} \frac{\lambda}{1+\lambda} \Phi^{\prime}(e) .
\end{aligned}
$$

It can be shown that $e_{e n v}^{I}<e_{\exp }^{I}$, for every $x \in[0, \bar{x})$. Hence, with experts a higher effort (more efficient) can be sustained. Both efforts are decreasing on $\lambda$ and $\left|\frac{\partial e_{\text {exp }}^{I}}{\partial \lambda}\right|>\left|\frac{\partial e_{\text {env }}^{I}}{\partial \lambda}\right|$. Hence as $\lambda$ increases, the efforts with experts and with environmentalists tend to converge, what vanishes the efficiency derived from using experts. This, together with the cost comparison ensures that there exists a $\overline{\bar{\lambda}}>\bar{\lambda}$, such that the optimal configuration for $x<\bar{x}$ is the same as if $x \geq \bar{x}$, only replacing $\bar{\lambda}$ by $\overline{\bar{\lambda}}$.

Remark 1 From here on we will make a change of variable that will be useful for the proofs: $\bar{\alpha}=\frac{1}{1-\beta}$, as $\bar{\alpha} \in(1,2]$, then $\beta \in\left(0, \frac{1}{2}\right]$. This change eases the proofs since it makes the deviation of the efficient firm $(\gamma)$, be linear in $\beta$, i.e. $\gamma=\beta$ e. 


\section{Proof of Proposition 5:}

The objective function of the regulator $W^{I}$ (disregarding the costs of acquiring the information), is given by:

$$
\begin{aligned}
& W^{I}=(1-x)\left(S-V\left(\widehat{\alpha}, e^{I}\right)-(1+\lambda) \Psi\left(e^{I}\right)\right)+ \\
x & {\left[v\left(S-V\left(\frac{1}{1-\beta}, \bar{e}\right)-(1+\lambda) \Psi(\bar{e})-\lambda \Phi\left(\max \left(e^{I}, \underline{e}\right)\right)\right)+(1-v)(S-V(1, \underline{e})-(1+\lambda) \Psi(\underline{e}))\right] . }
\end{aligned}
$$

Let us denote:

$$
\begin{gathered}
\bar{W}=S-V\left(\frac{1}{1-\beta}, \bar{e}\right)-(1+\lambda) \Psi(\bar{e}) \\
\underline{W}=S-V(1, \underline{e})-(1+\lambda) \Psi(\underline{e}) \\
\widetilde{W}=S-V\left(\widehat{\alpha}, e^{I}\right)-(1+\lambda) \Psi\left(e^{I}\right)
\end{gathered}
$$

With the Envelope Theorem, we compute the first order condition,

$$
\frac{\partial W^{I}}{\partial x}=v \bar{W}+(1-v) \underline{W}-\widetilde{W}-\lambda v \Phi\left(\max \left(e^{I}, \underline{e}\right)\right) .
$$

This difference is increasing in $x$, because:

1.- $v \bar{W}+(1-v) \underline{W}-\widetilde{W}$ is increasing in $x$ as this is the difference in surplus among the separating and the pooling contract. This difference is positive and increasing in $x$ because the distortion in the pooling level of effort $\left(e^{I}\right)$ is more important the higher is the value of $x$.

2.- $\lambda v \Phi\left(\max \left(e^{I}, \underline{e}\right)\right)$ is decreasing in $x$ because the informational rents are monotonically increasing in the effort levels and $\frac{\partial e^{I}}{\partial x}<0, \frac{\partial e}{\partial x} \leq 0$.

Hence the objective function is convex and we only have to compare the value functions at the extremes of the domain, for the given cost function, and prior about the types:

$$
\begin{aligned}
& W^{I}(x=0)=S-\left(D-\widehat{\alpha} e^{I}\right)-(1+\lambda) \frac{\left(e^{I}\right)^{2}}{2}=S-D+\frac{\widehat{\alpha}^{2}}{2(1+\lambda)} . \\
& W^{I}(x=1)=S-D+\frac{1}{2}\left[\underline{e}+\frac{\bar{e}}{1-\beta}-(1+\lambda)\left(\frac{(\bar{e})^{2}}{2}+\frac{(\underline{e})^{2}}{2}\right)-\lambda\left(\frac{(\underline{e})^{2} \beta(2-\beta)}{2}\right)\right] .
\end{aligned}
$$

Substituting the efforts:

$$
\begin{array}{llcc}
\text { For } x=0 & \bar{e}=\frac{1}{(1-\beta)(1+\lambda)} & \underline{e}=\frac{1}{(1+\lambda)} & e^{I}=\frac{(2-\beta)}{2(1+\lambda)(1-\beta)} \\
\text { For } x=1 & \bar{e}=\frac{1}{(1-\beta)(1+\lambda)} & \underline{e}=\frac{1}{1+\lambda(1+\beta(2-\beta))} & e^{I}=\frac{1}{1+\lambda(1+\beta(2-\beta))}
\end{array}
$$

and computing the difference we get:

$$
W^{I}(x=1)-W^{I}(x=0)=\frac{1}{2}\left[\frac{(1+\lambda(1+\beta(2-\beta)))\left(1-(2-\beta)^{2}\right)+(1-\beta)^{2}(1+\lambda)}{2(1-\beta)^{2}(1+\lambda)(1+\lambda(1+\beta(2-\beta)))}\right] .
$$

From here we get:

$$
W^{I}(x=1)>W^{I}(x=0) \Leftrightarrow \lambda<\frac{\beta}{4-11 \beta+6 \beta^{2}-\beta^{3}}=\lambda(\beta) .
$$


It can be shown that $\frac{\partial \lambda(\beta)}{\partial \beta}>0$, and that $\lim _{\beta \rightarrow \bar{\beta}} \lambda(\beta)=+\infty$, for $\bar{\beta}=0.47862$.

Undoing the change of variable, $\lambda(\bar{\alpha})=\frac{(\bar{\alpha}-1) \bar{\alpha}^{2}}{1+3 \bar{\alpha}+2 \bar{\alpha}^{2}-2 \bar{\alpha}^{3}}$, with $\lim _{\bar{\alpha} \rightarrow \bar{\alpha}_{\max }} \lambda(\bar{\alpha})=+\infty$ for $\bar{\alpha}_{\max }=1.918$.

\section{Proof of Proposition 6:}

We first provide a Lemma ensuring an interior solution in the problem of the principal.

Lemma: If $\lambda<\frac{2 \beta}{8-14 \beta+6 \beta^{2}+\beta^{3}}$, then $x^{R} \in(0,1)$.

Proof. As $C(0)=C^{\prime}(0)=0$, and $\lim _{x \rightarrow 1} C^{\prime}(x)=+\infty$, then a sufficient condition for $x^{R} \in(0,1)$ is ${\frac{\partial W^{I}}{\partial x}}_{\mid x=0}>0$.

$$
{\frac{\partial W^{I}}{\partial x}}_{\mid x=0}=v \bar{W}_{\mid x=0}+(1-v) \underline{W}_{\mid x=0}-\widetilde{W}_{\mid x=0}-\lambda v \Phi\left(e_{\mid x=0}^{I}\right)-C^{\prime}(0) .
$$

Substituting the effort levels and simplifying, it yields:

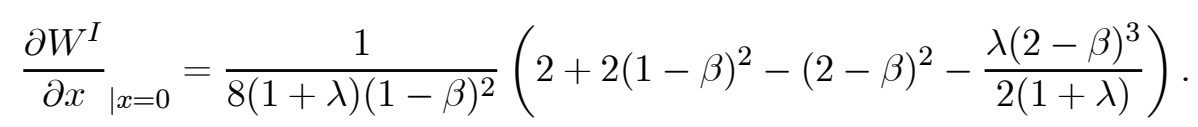

From here:

$$
{\frac{\partial W^{I}}{\partial x}}_{\mid x=0}>0 \Longleftrightarrow \lambda<\frac{2 \beta}{8-14 \beta+6 \beta^{2}+\beta^{3}} .
$$

We start the proof of the Proposition with the program of the firm, its expected profits are:

$$
E \Pi(x)=x v \Phi\left(\max \left(e^{I}, \underline{e}\right)\right)-C(x) .
$$

As the value of $x$ is chosen simultaneously to the design of the contract, the effort levels are taken as given by the firm. The associated first order condition is:

$$
\frac{\partial E \Pi}{\partial x}=v \Phi\left(\max \left(e^{I}, \underline{e}\right)\right)-C^{\prime}(x) \geq 0
$$

The assumptions on $C(x)$ ensure that the optimal choice of the firm $\left(x^{F}\right)$ is always interior.

For the program of the principal, we can take some intermediate results of the proof of Proposition 5. In particular, the first order condition of the regulator's objective function, with respect to $x$ including the costs is:

$$
\frac{\partial W^{I}}{\partial x}=v \bar{W}+(1-v) \underline{W}-\widetilde{W}-\lambda v \Phi\left(\max \left(e^{I}, \underline{e}\right)\right)-C^{\prime}(x) .
$$

The difference of the two first order conditions is:

$$
\frac{\partial W^{I}}{\partial x}-\frac{\partial E \Pi}{\partial x}=v \bar{W}+(1-v) \underline{W}-\widetilde{W}-(1+\lambda) v \Phi\left(\max \left(e^{I}, \underline{e}\right)\right) .
$$

By the argument constructed in the previous proof we know that this difference is increasing in $x$. We only need to evaluate $\frac{\partial W^{I}}{\partial x}-\frac{\partial E \Pi}{\partial x}$ in the extreme values of $x$, for $\Psi(e)=\frac{e^{2}}{2}$, and $v=\frac{1}{2}$.

For $x=1,\left(\frac{\partial W^{I}}{\partial x}-\frac{\partial E \Pi}{\partial x}\right)_{\mid x=1}=\frac{1}{2}\left[\underline{e}(1-2 \widehat{\alpha})+\frac{1}{1-\beta} \bar{e}-(1+\lambda)\left(\frac{\bar{e}^{2}}{2}-\frac{e^{2}}{2}(1-\beta(2-\beta))\right]\right.$.

Substituting the effort levels, we get: 
$\left(\frac{\partial W^{I}}{\partial x}-\frac{\partial E \Pi}{\partial x}\right)_{\mid x=1}=\frac{1}{2}\left[\frac{1}{2(1-\beta)^{2}(1+\lambda)}-\frac{2 \beta(1+\lambda(1+\beta(2-\beta))-(1+\lambda)(1-\beta(2-\beta))(1-\beta)}{2\left(1+\lambda(1+\beta(2-\beta))^{2}(1-\beta)\right.}\right]$.

It can be shown that $\left(\frac{\partial W^{I}}{\partial x}-\frac{\partial E \Pi}{\partial x}\right)_{\mid x=1}>0, \forall \lambda>0, \forall \beta \in\left(0, \frac{1}{2}\right)$.

For $x=0$, and proceeding analogously, we find:

$\left(\frac{\partial W^{I}}{\partial x}-\frac{\partial E \Pi}{\partial x}\right)_{\mid x=0}=\frac{-4(1+\lambda)(1-\beta)+4\left(1-\frac{\beta}{2}\right)^{2}\left(1+\lambda(1-\beta)^{2}\right)+\beta\left(\beta(1+\lambda)-(2-\beta)^{3}\right)}{16(1-\beta)^{2}(1+\lambda)^{2}}$.

The $\operatorname{sign}\left[\left(\frac{\partial W^{I}}{\partial x}-\frac{\partial E \Pi}{\partial x}\right)_{\mid x=0}\right]=\operatorname{sign}\left[\beta^{3}-6 \beta^{2}+14 \beta-8\right]<0, \forall \lambda>0, \forall \beta \in\left(0, \frac{1}{2}\right)$.

Therefore if the condition in the Lemma holds, we know:

$$
\begin{gathered}
0<\frac{\partial W^{I}}{\partial x}(x=0)<\frac{\partial E \Pi}{\partial x}(x=0) \\
0>\frac{\partial W^{I}}{\partial x}(x=1)>\frac{\partial E \Pi}{\partial x}(x=1) \\
\frac{\partial}{\partial x}\left(\frac{\partial W^{I}}{\partial x}-\frac{\partial E \Pi}{\partial x}\right)>0 .
\end{gathered}
$$

\section{Proof of Proposition 7:}

We need to study independently two regions, $x \leq \bar{x}=\frac{1+\lambda}{1+\lambda(1+(1-\beta)(2-\beta))}$, and $x>\bar{x}$.

For $x \leq \bar{x}$,

$$
\frac{\partial^{2} W^{I}}{\partial x \partial \lambda}=-\left(\frac{\left((1-\beta) e^{I}\right)^{2}}{2}+\frac{1}{2} \frac{\bar{e}^{2}}{2}+\frac{\bar{e}^{2}}{2}-\frac{1}{2} \frac{e^{2}}{2}\right)-\frac{\partial e^{I}}{\partial \lambda}(1+\lambda)\left(\frac{2-\beta}{2(1-\beta)(1+\lambda)}+\frac{\lambda \beta(2-\beta) e^{I}}{2(1+\lambda)}-e^{I}\right)
$$

Substituting the effort levels by the optimal values, and simplifying, yields:

$$
\frac{\partial^{2} W^{I}}{\partial x \partial \lambda}=\frac{-1}{2}\left[\left(\frac{(2-\beta)(1-x)}{2(1+\lambda)(1-x)+\lambda x \beta(2-\beta)}\right)^{2}+\frac{1+(1-\beta)^{2}}{2(1-\beta)^{2}(1+\lambda)^{2}}-\frac{\lambda \beta(2-\beta)^{3}(1-x)(2(1-x)-x \beta(2-\beta))}{(1-\beta)^{2}(2(1+\lambda)(1-x)+\lambda x \beta(2-\beta))^{3}}\right]
$$

Proceeding analogously for the region $x>\bar{x}$, we find:

$$
\begin{aligned}
\frac{\partial^{2} W^{I}}{\partial x \partial \lambda}=\frac{-1}{2}\left[\frac{1}{2(1-\beta)^{2}(1+\lambda)^{2}}\right. & \left.+\frac{(2-\beta-x)^{2}}{((1+\lambda)(2-x)+\lambda x \beta(2-\beta))^{2}}\right] \\
& +\frac{(2-\beta-x)(2-x+x \beta(2-\beta)) \beta}{2((1+\lambda)(2-x)+\lambda x \beta(2-\beta))(1-\beta)^{2}(2-x)}\left(1+\frac{\lambda(2-\beta)(2-\beta-x)}{(1+\lambda)(2-x)+\lambda x \beta(2-\beta)}\right)
\end{aligned}
$$

Due to the impossibility to algebraically obtain the sign of these two expressions, we make use of numerical calculations. These calculations show that, $\forall \beta \in\left(0, \frac{1}{2}\right), \forall x \in(0,1)$ and $\forall \lambda \in$ $(0,2):^{15}$

$$
\frac{\partial^{2} W^{I}}{\partial x \partial \lambda}<0
$$

\section{Proof of Lemma 2:}

\footnotetext{
${ }^{15}$ We have restricted the domain of $\lambda$ in order to be able to perform the numerical computations. In the restricted domain we use, we let the public expenditures have a real cost up to three times its nominal one.
} 
We have to compare the expected profits of the firm under the alternative timings, these are given by:

$$
\begin{aligned}
E \Pi_{e n-f}(x) & =x \widetilde{v} \Phi\left(\max \left(e^{I}, \underline{e}\right)\right)-C(x) \\
E \Pi_{e n-c}(x) & =x v(1-z) \Phi\left(\max \left(e^{I}, \underline{e}\right)\right)-C(x) \\
E \Pi_{e x-f}(x) & =x v \Phi\left(\max \left(e^{I}, \underline{e}\right)\right)-C(x) \\
E \Pi_{e x-c}(x) & =x v(1-z) \Phi\left(\max \left(e^{I}, \underline{e}\right)\right)-C(x) .
\end{aligned}
$$

From here it is straightforward to rank the first order conditions and obtain that:

$$
x_{e x-f}^{F}>x_{e n-f}^{F}>x_{e n-c}^{F}=x_{e x-c .}^{F} .
$$




\section{References}

[1] Boyer, M. and Laffont, J. J. (1999) "Toward a Political Theory of the Emergence of Environmental Incentive Regulation". Rand Journal of Economics, 30, 137-157.

[2] Council of the European Communities. (1985) Council Directive 85/337/EEC of 27 June 1985 on the assessment of the effects of certain public and private projects on the environment.

[3] Council of the European Union. (1997) Council Directive 97/11/EC of 3 March 1997 amending Directive 85/337/EEC on the assessment of the effects of certain public and private projects on the environment.

[4] Crémer, J. and Khalil, F. (1994) "Gathering Information Before the Contract is Offered: The Case with Two States of Nature". European Economic Review, 38, 675-682.

[5] Crémer, J. and Khalil, F. (1998) "Strategic Information Gathering Before a Contract is Offered". Journal of Economic Theory. 81, 163-200.

[6] Dewatripont, M. and Tirole, J. (1999) "Advocates". Journal of Political Economy. 107-1, $1-39$.

[7] Jones, L. P., Tandon, P. and Vogelsand, I. (1990) Selling Public Enterprises. Cambridge, Mass. MIT Press.

[8] Kessler, A. S. (1998) "The Value of Ignorance". Rand Journal of Economics. 29-2, 339-354.

[9] Laffont, J. J. and Tirole, J. (1991) "The Politics of Government Decision Making: A Theory of Regulatory Capture". The Quarterly Journal of Economics, 1089-1127.

[10] Laffont, J. J. and Tirole, J. (1993) A Theory of Incentives in Procurement and Regulation. Cambridge, Mass. MIT Press.

[11] Lewis, T. R., (1996) "Protecting the Environment when Costs and Benefits are Privately Known". Rand Journal of Economics. 27-4, 819-847.

[12] Lewis, T. R. and Sappington, D. E. M. (1993). "Ignorance in Agency Problems". Journal of Economic Theory. 61, 169-183.

[13] Milgrom, P. and Roberts, J. (1986) "Relying on the Information of Interested Parties". Rand Journal of Economics. 17-1, 18-32. 


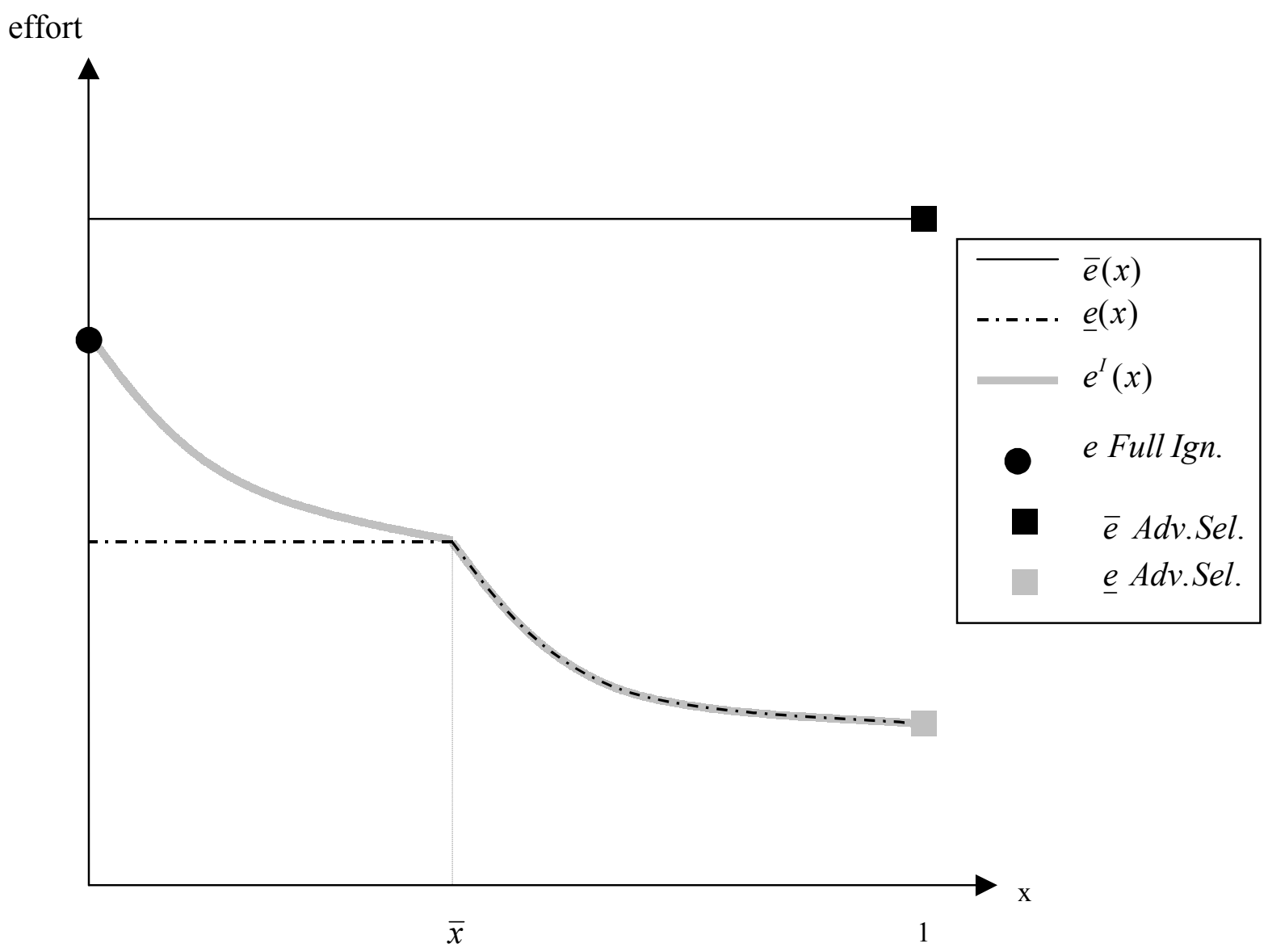

Figure 1: Optimal Menu of Contracts as a Function of $\mathrm{x}$ 